\title{
The impact of turbidity on foraging and risk taking in the invasive Nile tilapia (Oreochromis niloticus) and a threatened native cichlid (Oreochromis amphimelas)
}

\author{
Jonathan D. B. Wing ${ }^{1}$ (D) $\cdot$ Toby S. Champneys $^{1}$ (I) $\cdot$ Christos C. Ioannou ${ }^{1}$ (I) \\ Received: 22 July 2020 / Revised: 14 January 2021 / Accepted: 20 January 2021 / Published online: 10 February 2021 \\ (C) The Author(s) 2021
}

\begin{abstract}
Anthropogenic activity can increase water turbidity, changing fish behaviour by reducing visibility. The spread of invasive species is also facilitated by human activity, further increasing the pressure on native species. In two experiments, we measured the foraging efficiency, risk perception and inter-individual consistency of risk-taking (personality variation in boldness) of an invasive species, the Nile tilapia (Oreochromis niloticus), and a threatened tilapia, the Manyara tilapia (Oreochromis amphimelas), in clear and turbid water. In experiment one, O. niloticus was faster to initiate feeding, encountered more food items and consumed more than $O$. amphimelas. The latency to start foraging by $O$. niloticus decreased in turbid water. Turbidity did not affect the latency to start foraging in $O$. amphimelas but the number of food items they encountered was highest at the intermediate turbidity. There was however no significant effect of turbidity in either species on the total food consumed. In contrast to this foraging context, in experiment two with a refuge and no food available, risk taking behaviour was similar in both species and they both responded with similarly reduced risk taking in turbid water. Evidence of personality variation was weak, being observed only in $O$. amphimelas when first leaving the shelter in turbid water. Overall, species differences were greater in the foraging context but turbidity was more important in the risk-taking context. O. amphimelas is more sensitive to turbidity during foraging, and $O$. niloticus is likely to have a competitive advantage in foraging situations, especially in degraded turbid habitats.
\end{abstract}

\section{Significance statement}

Under human-induced environmental change, native species are often exposed to multiple stressors. Here, we tested the responses of two cichlid fish to increasing turbidity, the Nile tilapia (Oreochromis niloticus), which is invasive throughout the tropics, and the Manyara tilapia (Oreochromis amphimelas), a threatened species, indigenous to Tanzania. We found that turbidity was beneficial to the foraging of $O$. niloticus, which in both clear and turbid water consumed and encountered more food than $O$. amphimelas. In contrast, without food present, both species displayed similar responses of increased risk perception in turbid water with little evidence of personality variation between individuals in either species. Our results suggest that invasive species tolerant of degraded habitats may outcompete less well-adapted native species for food.

Keywords Boldness $\cdot$ Personality variation $\cdot$ Habitat degradation $\cdot$ Environmental stressors $\cdot$ Multiple stressors $\cdot$ Invasive species

Communicated by K. Lindström

Jonathan D. B. Wing

jon.dbwing@protonmail.com

1 School of Biological Sciences, University of Bristol, Life Sciences Building, 24 Tyndall Avenue, Bristol BS8 1TQ, UK

\section{Introduction}

Turbidity is the cloudiness of water produced by suspended particles scattering light (Davies-Colley and Smith 2001). This alters the contrast between an object and its background, influencing the vision of aquatic organisms (Utne-Palm 2002). Many high productivity aquatic habitats are naturally turbid and are populated by species which are specifically adapted to these environments (Williams 1982; Fabricius et al. 2005; Van De Meutter et al. 2005; Wenger et al. 
2011). However, numerous anthropogenic activities can rapidly increase turbidity above natural levels, resulting in up to a 10-fold increase in sediment loading (Hilton and Phillips 1982; Dearing and Jones 2003; Järvenpää and Lindström 2004; Erftemeijer et al. 2012). Human-induced eutrophication and increased sedimentation are growing causes of habitat degradation in clear water systems and can drive rapid changes to the sensory environment (Julien 1995; Mol and Ouboter 2004; Dodds et al. 2009; Erftemeijer et al. 2012). Such changes influence the behaviour of many fish species that rely on vision, especially during foraging and predator-prey interactions (Guthrie and Muntz 1986; Abrahams and Kattenfeld 1997; Ferrari et al. 2010a; Wishingrad et al. 2015; Ehlman et al. 2020). Additionally, turbidity can influence many other functions related to reproductive success, including sexual selection, mate choice and parental care (Järvenpää and Lindström 2004, 2011). As a result, the behavioural responses of species to increased turbidity can heavily influence the effect it has on the survival of individuals and populations (Caves et al. 2017).

The effect of turbidity on foraging efficiency varies across species and ecological contexts. In some cases, turbidity can increase the contrast of an object against its background, benefiting object detection and improving foraging (Hinshaw 1985). More generally however, turbid water has been shown to restrict the visual range of fish and reduce the efficiency of visual foragers (Utne-Palm 2002; Lunt and Smee 2015). This negatively affects prey detection, reducing the reaction distance within which predators can detect their prey (Barrett et al. 1992; Gregory 1993; Sweka and Hartman 2003; Quesenberry et al. 2007). Therefore, foraging success can be constrained even in areas of high prey density (Turesson and Brönmark 2007; Becker et al. 2016; Snow et al. 2018). This has been demonstrated in brown trout (Salmo trutta) and bluegill sunfish (Lepomis macrochirus) where foraging performance and reactive distance declined as turbidity increased (Vinyard and O'brien 1976; Stuart-Smith et al. 2004). Even after initial detection, turbidity may impact foraging, with some species displaying reduced attack success even at low levels of turbidity (8 Nephelometric Turbidity Units (NTU)) (Johansen and Jones 2013). A common behavioural response to reduced visibility is to increase activity, as it can increase encounter rates (Sweka and Hartman 2001; Granqvist and Mattila 2004; Harvey and White 2008). Despite this, such behaviours are potentially detrimental if improved encounter rates do not compensate for the increased cost in energy or the greater risk of encountering predators (Meager and Batty 2007).

Turbid environments also influence predator-prey interactions by changing the perception of risk in fish (Gregory 1993; Chamberlain and Ioannou 2019; Ehlman et al. 2019). Prey may use turbid conditions as refugia from predators, where they are less likely to be detected (Engström-Öst et al. 2009).
Chinook salmon (Oncorhynchus tshawytscha), for example, reduce antipredator behaviour in turbid conditions, suggesting a decreased perception of risk (Gregory 1993). However, this can be maladaptive if the true risk of predation does not decrease (Abrahams and Kattenfeld 1997; Lehtiniemi et al. 2005). In other cases, fish may reduce activity and feeding while increasing shelter use in turbid areas, indicating an increase in risk perception (Leahy et al. 2011; Ajemian et al. 2015). Additionally, turbidity can impair the ability of some fish to learn about and to recognise predators, slowing the reaction time of prey (Ferrari et al. 2010b; Chivers et al. 2013).

Changes to foraging efficiency and risk perception due to turbidity thus impact the fitness of fish species (Tuomainen and Candolin 2011). However, individuals within a population may react differently to similar stimuli and this interindividual consistency of behaviour is referred to as animal temperament or personality variation (Réale et al. 2007). A behavioural trait that is often consistently different between individuals is boldness, defined by the likelihood of an individual to take risks in return of greater rewards (Réale et al. 2007). The repeatability of behaviours associated with boldness can be induced by the combined effect of turbidity and the presence of predators (Ehlman et al. 2019). As personality variation indicates a lack of behavioural plasticity, it suggests that individuals may be constrained in their response to environmental change (Tuomainen and Candolin 2011).

In addition to turbidity, the introduction of non-native species poses a great threat to aquatic ecosystems, reducing biodiversity by displacing native species and contributing to habitat degradation (Mainka and Howard 2010). The casual mechanisms underlying the success and negative effects of many invasive species are, however, poorly understood (MacDougall and Turkington 2005; Tuomainen and Candolin 2011; Gallardo et al. 2016). Improving understanding of these mechanisms is an important goal of invasive species research, as it is crucial in making informed decisions regarding environmental management, and when allocating limited resources to the prevention of the most harmful species (Vander Zanden and Olden 2008). Successful invasive species often exhibit tolerance to a wide range of environmental conditions and adapt quickly to novel environments. This characteristic is so prevalent that the presence of invasive species has been suggested as an indicator of habitat degradation ( Kennard et al. 2005; García-Berthou 2007). Improved tolerance of degraded environments can provide a competitive advantage for non-native species, allowing effective colonisation as intolerant native species decline (MacDougall and Turkington 2005; Linde et al. 2008a). For example, the combination of turbidity and the presence of the invasive yellowfin shiner (Notropis lutipinnis) reduces the feeding efficiency of the rosyside dace (Clinostomus funduloides) through the combined effects of restricted reaction distance and interspecific competition (Hazelton and Grossman 
2009a, b). Resident species are often exposed to multiple stressor effects from anthropogenic activity (Ormerod et al. 2010; Orr et al. 2020), which can have a greater combined effect than each stressor in isolation (i.e. a synergistic effect).

The Nile tilapia (Oreochromis niloticus) is a widespread invasive cichlid species commonly used in aquaculture. Subsequently, non-native populations of $O$. niloticus have been established across the tropics (Canonico et al. 2005; Mohamed and M Al-Wan 2020). Like many invasive species, $O$. niloticus shows a high tolerance to a wide range of environmental conditions. For example, juvenile $O$. niloticus abundance has been shown to increase in degraded habitats, while the abundance of native species declines (Linde et al. 2008b). Additionally, O. niloticus can actively increase turbidity through benthic foraging, by resuspending sediment and increasing nutrient levels leading to algal growth (Zhang et al. 2017).

In this experiment, we investigated the behavioural responses of $O$. niloticus and the Manyara tilapia Oreochromis amphimelas in clear compared to turbid water. O. amphimelas is a threatened tilapia species native to Tanzania where it lives in sympatry with the invasive $O$. niloticus across five lakes (Shechonge et al. 2019). In both species, we measured how foraging efficiency changes across low, medium and high turbidity ( 0,15 and $30 \mathrm{NTU})$, and whether risk taking and consistent inter-individual differences in risk taking (i.e. personality variation in boldness) were different in clear and turbid treatments ( 0 and $15 \mathrm{NTU}$ ). Interactions between multiple stressors can be synergistic or antagonistic, either limiting or exacerbating the harmful impacts they have on affected ecosystems (Piggott et al. 2015). Therefore, investigating the relationship between turbidity and the behaviour of native and invasive fish species could provide useful insights into how native species may be impacted by two widespread ecological stressors with the potential to interact.

\section{Materials and methods}

\section{Subjects}

Sixty-eight $O$. amphimelas $(65.3 \pm 7.5 \mathrm{~mm}$ mean \pm SD body length) were bred from first-generation wild caught stock by Bangor University and transported to Bristol in December 2018. Sixty-eight $O$. niloticus $(79.6 \pm 6.9 \mathrm{~mm}$ mean $\pm \mathrm{SD}$ body length) were purchased from Fish Farm UK, London, in December 2018. Thirty-six O. amphimelas and $36 O$. niloticus were used in experiment one and 29 O. amphimelas and $32 \mathrm{O}$. niloticus were used in experiment two. Individuals were not reused between the two experiments and fish were housed in separate tanks according to the experiment they participated in. Three $O$. amphimelas were removed during the second experiment due to ill health during the trial period.
The two species were housed separately in clear water $(0$ NTU) within a recirculating aquarium system in $180 \mathrm{~L}$ glass tanks. Plastic plants and pipes were placed in the housing tanks to provide enrichment (Brydges and Braithwaite 2009). Identical shelters used in the experiments were also placed in the housing tanks to reduce novelty in the experimental trials (Fig. 1c). Fish were fed daily with a diet consisting of ZM large Premium Granular feed (Techniplast, London, UK), TetraMin flake (Tetra, Melle, Germany), frozen bloodworm (CC Moore \& Co, Templecoombe, UK) and Gamma $^{\text {TM }}$ Krill Pacifca, chopped prawn, Mysis shrimp, brine shrimp and vegetable diet (Tropical Marine Centre, Chorleywood, UK). Water temperature in the housing tanks was sustained at $26^{\circ} \mathrm{C}$ with a constant diurnal light cycle of 12:12 h (light:dark) mimicking tropical conditions. All fish remained in the laboratory for future use following the experiments.

\section{Experimental setup}

The trials were conducted in a white acrylic tank with an opaque divider creating two arenas (each $80.5 \times 64 \times 40 \mathrm{~cm}$, width $\times$ length $\times$ height, containing $77.2 \mathrm{~L}$ of water at a depth of $15 \mathrm{~cm}$ ) so that two trials could be conducted simultaneously (Fig. 1a). A shelter created from plastic ornamental plants attached to a white corrugated PVC base was placed in each side of the tank to act as a shelter in experiment two (Fig. 1c). Turbidity was recorded with a calibrated Thermo Scientific ${ }^{\mathrm{TM}}$ Orion ${ }^{\mathrm{TM}}$ AQUAfast AQ3010 turbidity meter using nephelometric turbidity units (NTU). The tank was lit from above with a fluorescent strip light and recorded from above with a GoPro Hero 5 Black (GoPro Inc.) at a resolution of $1920 \times 1080$ pixels. The tank was filled with water from the recirculation system used to house the fish. A white curtain was drawn across the tank area to prevent disturbance.

\section{Procedure}

Experiment one: the effect of turbidity on foraging efficiency

Each day of testing, one of three turbidity levels (0, 15 and 30 NTU) was randomly selected and 12 individuals (six of each species) were tested. Kaolin clay was used to create treatments of $15 \mathrm{NTU}(0.04 \mathrm{mg} / \mathrm{l})$ and $30 \mathrm{NTU}(0.09 \mathrm{mg} / \mathrm{l})$. The clay powder was spread evenly across the tank and mixed until the desired level of turbidity was attained following previous studies (Quesenberry et al. 2007; Leahy et al. 2011). The turbidity range used was similar to levels in other studies, staying below the upper tolerance levels of fish and not interfering with the transmission of chemical cues or altering the $\mathrm{pH}$ or hardness of the water (Horppila et al. 2004; Leahy et al. 2011). Anthropogenic activities such as mining can result in 
a

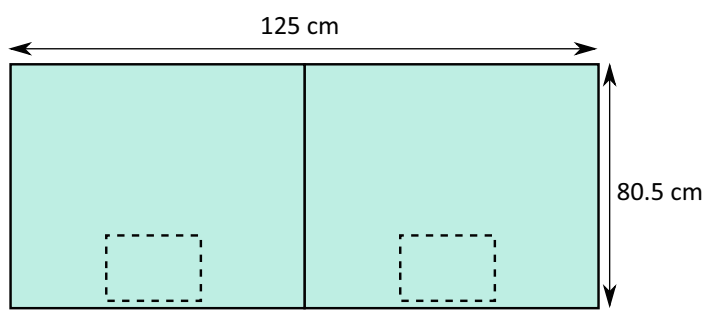

c

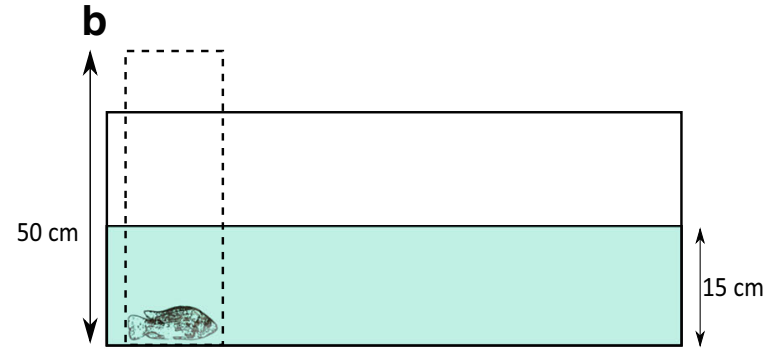

d

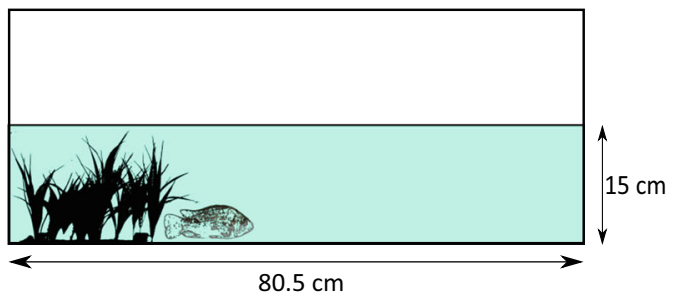

Fig. 1 The experimental arena and shelter used in both experiments (not to scale). a Top-down view of the experimental arena and the position of the acclimation boxes used in experiment one (dashed). $\mathbf{b}$ Side view of the trial arena, with the removable acclimation box (dashed). $\mathbf{c}$ The plant shelter used in experiment two, made from plastic aquarium plants fixed to a plastic base and weighted with ornamental rocks. $\mathbf{d}$ Side view of the trial arena showing the position of the plant shelter used in experiment two where the fish were placed at the beginning of the trial increasing levels of clay entering aquatic systems, making clay an ecologically relevant way of replicating natural levels of turbidity (Eriksson et al. 2004; Kemp et al. 2011). Resuspension by stirring was conducted between trials to ensure that the turbidity remained at desired levels across all trials.

The day before each trial, 6 individuals of each species were caught haphazardly with a hand net from their housing tanks and acclimatised to the next day's turbidity $(0,15$ or 30 NTU) in the trial tank for $16 \mathrm{~h}$ (overnight) prior to testing. The two species were acclimated separately in the two halves of the tank, separated by the opaque divider. Fish were not fed the day before the experiment to encourage feeding and standardise hunger and motivation. Before the first trial on each day, all fish were removed and kept in covered plastic holding tanks $(45 \times 32 \times 25 \mathrm{~cm}$, water depth $15 \mathrm{~cm})$ separated by species and with a turbidity matching that day's treatment. One $O$. niloticus and one $O$. amphimelas were netted, their total body length measured (mouth to the end of caudal fin) with digital callipers and were placed in an acclimation box in the trial area for $30 \mathrm{~min}$ before trials began (Fig. 1b). After this period, 10 pellets were added to each side of the experimental arena, spread evenly across the width opposite to the acclimation boxes. Floating pellets were used as food items (Hikari Cichlid Gold mini-pellet, $3.2-3.7 \mathrm{~mm}$ ) as they remained at the water surface and feeding attempts could be easily observed even in turbid conditions. Recording began and the acclimation boxes were slowly lifted and removed, releasing the fish. Trials lasted $15 \mathrm{~min}$. Each fish was tested once and then placed into housing tanks reserved for fish that had completed the trials to avoid repeated testing of the same individual. The arenas and holding tanks were emptied after each day of trials and replaced with fresh system water which was dosed with the desired turbidity for the next day. The next group of fish was then acclimated for the next day's trial. Trials took place between 30.04.2019 and 14.05.2019.

Video recordings were analysed using BORIS version 7.7.3 by a single reviewer to ensure consistency (Friard and Gamba 2016). The variables measured were the time to first feed, the number of feeding attempts (the number of the 10 pellets that the fish attempted to feed on) and the number of pellets consumed. A feeding attempt was defined as an individual's mouth making contact with a pellet at least once, so that subsequent attempts to feed on that pellet were not further counted. The number of feeding attempts thus measures the number of different food items that were encountered during the trial. It was not possible to record data blind as individuals had to be identified by species for each experiment.

\section{Experiment two: the effect of turbidity on risk taking and personality variation}

Consistent inter-individual differences in responses to turbidity were tested by exposing each fish to each treatment $(0$ and 15 NTU) twice across a 4-day period. Eight individuals of each species were tested per week and every individual took part in one trial per day for four consecutive days. Turbid water was created following the methods outlined in experiment one. The day before each 4-day trial period, 8 individuals of each species were caught haphazardly with a hand net. 
Fish were split into 4 groups (2 groups of $O$. niloticus and 2 groups of $O$. amphimelas) and the total body length of each fish was measured (mouth to the end of caudal fin) using digital callipers. Each group was housed in a plastic holding tank $(45 \times 32 \times 25 \mathrm{~cm}$, water depth $15 \mathrm{~cm})$, which were continuously oxygenated with air stones, for the next 4 days. These holding tanks were filled with system water which was changed each day with a turbidity level to match the next day's trials (0 or 15 NTU), using the methods outlined in the previous experiment, allowing the fish to be acclimated for $16 \mathrm{~h}$ (overnight) before the experiments began in the required treatment. The fish in each holding tank were different lengths to ensure clear identification of each individual was possible across the 4-day period. Trials took place between 18.06.2019 and the 26.07.2019.

The order of testing for groups, and individuals within groups, was allocated randomly per day. The water was stirred before each trial to maintain turbidity levels. Before each trial, the turbidity was measured to make sure it was at the desired level ( 0 or 15 NTU) or resuspended by stirring if needed. Video recording began and individuals were placed within the shelters (Fig. 1c, d). Trials lasted for $30 \mathrm{~min}$. Once tested, fish were returned to their holding tank. To standardise hunger levels between trials, fish were only fed after all individuals had taken part in the day's experiments. At the end of the 4day trial period, test fish were removed from the holding tanks and kept separately to untested fish to avoid individuals being used more than once. BORIS version 7.7.3 software (Friard and Gamba 2016) was used to analyse the videos by a single reviewer to maintain consistency. The variables measured were the time to first leave the shelter, the time taken to first cross the midline of the tank after first leaving the shelter and the total time spent out of the shelter. It was not possible to record data blind because our study required individuals to be identified across repeat experiments.

\section{Statistical analysis-experiment one}

$R$ version 3.5.3 was used for the analysis of both experiments $(R$ Core Team 2019). The time to first feeding attempt, a censored time-to-event response variable, was analysed using a Cox proportional hazard test. The explanatory variables included for this analysis were an interaction term between species and turbidity and their main effects, along with body length (a continuous variable). This test analyses how the likelihood of an event, in this case attempting to feed, changes depending on a set of risk factors, which in this case are the explanatory variables. The principal assumption of this test is that the hazards are proportional, meaning that the effect of explanatory variables do not change over time. The proportional hazards assumption was tested using Schoenfeld residuals (from R function 'cox.zph' in the 'survival' package) (Therneau 2015). This function provides $P$ values for each explanatory variable, with significant values meaning that the effect is not proportional over time, violating the assumption. We found no violations of this assumption based on confidence limits of 0.95 . To test the assumption of nonlinearity, models were run with a smoothing parameter fitted to the linear covariate body length using a penalised spline method (from function 'pspline' in the 'survival' package). Models with and without spline terms were then compared using AICc (Akaike information criterion corrected (AICc) for small sample sizes) with the function 'AICctab' in the 'bbmle' package (Bolker and R Core Team 2017). Models without spline terms were more likely indicating that a linear relationship between the time to first feeding attempt and body length. The deviance residuals were used to examine influential observations (from $\mathrm{R}$ function 'ggcoxdiagnostics', in the 'survminer' package) (Kassambara et al. 2019).

The number of feeding attempts was analysed with negative binomial generalised linear models (GLMs). The continuous variables were scaled in all models ( $\mathrm{R}$ function 'scale'). This provides a standardisation of the variable by first calculating the mean and standard deviation of all values in the vector, then subtracting the mean from each value and dividing each value by the standard deviation. The overdispersion statistic was calculated to ensure it was between 0.5 and 2 indicating no under or overdispersion. The model for the number of feeding attempts initially included an interaction term between species and turbidity and their main effects, along with a continuous variable (body length), and a categorical variable (the side of the arena that the trial took place in). After observing a nonlinear relationship between turbidity and the number of feeding attempts, polynomial regression was applied to provide a nonlinear fit to the model ( $\mathrm{R}$ function 'poly') (Becker et al. 1989; James et al. 2000; Chambers 2017). Likelihood ratio tests were used to remove any nonsignificant interactions or explanatory variables by deleting terms based on likelihood ratio tests (using R function 'drop1' in the 'Ime4' package) (Bates et al. 2015).

The number of pellets consumed was analysed with negative binomial GLMs. These models initially included an interaction term between species and turbidity and their main effects, along with a continuous variable (body length), and a categorical variable (the side of the arena that the trial took place in). Continuous variables were scaled and assumptions were tested using the same methods as outlined for the number of feeding attempts. Once again, the 'drop 1' function was used to remove nonsignificant interactions and explanatory variables.

\section{Statistical analysis-experiment two}

The time taken to first leave the shelter and the time taken to first cross the midline of the arena, censored time-to-event response variables, were analysed using Cox proportional hazards models. The explanatory variables included for this analysis were an interaction term between species and 
turbidity and their main effects, along with a continuous variable (body length). The same methods as outlined in experiment one were used to test for proportional hazards, nonlinearity and influential observations. We found that models including spline terms fitted to the linear covariate body length were more likely for both the time taken to leave shelter and the time to cross the midline. This suggests that body length had a non-linear effect on these dependent variables. Smoothing penalised splines were therefore added to improve model fit. Once spline terms were added, we found no evidence for violations of any of the test assumptions.

The total time spent outside the shelter was analysed with a generalised linear mixed model (GLMM) with a Poisson distribution using the default 'log' link function. The model included an interaction between species and turbidity and their main effects, a continuous variable (body length) and a categorical variable (the side of the arena that the trial took place in) and a random effect (fish ID). To reduce overdispersion, continuous variables were scaled and an observation-level random effect term was included in the model (Harrison 2014). Likelihood ratio tests were then used to remove nonsignificant interactions by deleting terms based on chi squared tests using the 'drop 1' function.

To assess consistency in inter-individual variation (i.e. personality variation) for each of the three response variables, in each species and in each of the turbidity treatment separately, Spearman rank non-parametric correlations were used. ShapiroWilk normality tests showed that the data was not normally distributed $(P<0.05)$ and the censored data for the two latency response variables can lead to spurious estimates of formal repeatability scores (Stamps et al. 2012; Ioannou and Dall 2016).

Given that the body length distribution differed between the two species, variance inflation factors were used to test for multicollinearity in fixed effects across all Cox proportional hazards models and generalized linear models in both experiments (using the 'vif' function in the 'car' package, Fox and Weisberg 2019). We found evidence for low multicollinearity between explanatory variables, with all vif values being below 5 .

Finally, given that sampling using a hand net could result in a bias towards the boldest individuals first (Biro and Dingemanse 2009), we conducted a negative binomial GLM including the time spent outside of the shelter as the response variable and how early the fish were caught (experiment block number 1-16) as the explanatory variable.

\section{Results}

\section{Experiment one}

A significant interaction between species and turbidity revealed that turbidity had a different effect on the likelihood of a first feeding attempt between the two species (Cox proportional hazard model $(\mathrm{CPH})$ : hazard ratio $(\mathrm{HZ})=0.95, N=$ 72, $P<0.046$ ). This, along with the trend shown in Fig. 2 , suggests that in $O$. amphimelas turbidity had no effect on the time taken to first attempt to feed, while in $O$. niloticus, the first feed attempt was more likely to occur at 15 and 30 NTU than it was at 0 NTU (Fig. 2a, b).

The explanatory variables included for the analysis of the number of food items that the fish attempted to feed on were an interaction term between species and turbidity and their main effects, along with a continuous variable (body length). When this model was restricted to fitting a linear relationship between turbidity and the response variable, there was no significant interaction between species and turbidity (negative binomial GLM: species * (scale(turbidity)): $\mathrm{LRT}_{1,66}=0.02, P$ $=0.88$ ) or a linear effect of turbidity (negative binomial GLM: (scale(turbidity)): $\mathrm{LRT}_{1,67}=1.18, P=0.27$ ). The effect of species was significant; $O$. niloticus attempted to feed on a greater number of food items (negative binomial GLM:
Fig. 2 Kaplan-Meier estimates of the proportion of fish that did not feed across the trial period (a) $O$. amphimelas and (b) O. niloticus in 0 (black), 15 (light blue) and 30 (dark blue) NTU (turbidity level: nephelometric turbidity units) treatments a

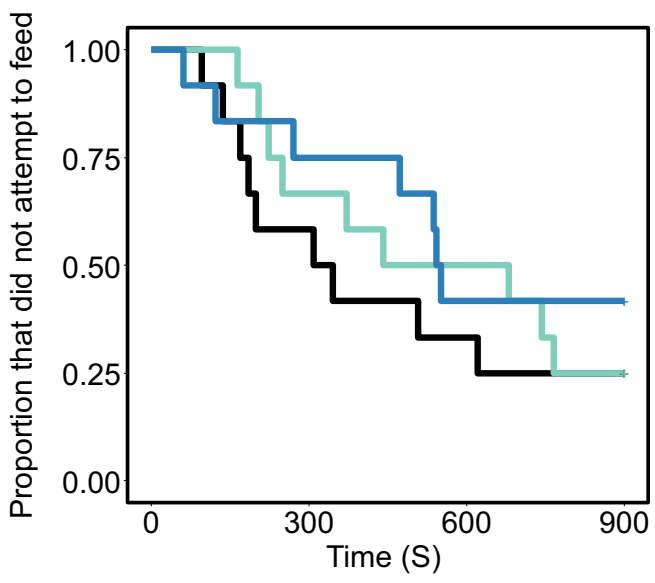

b

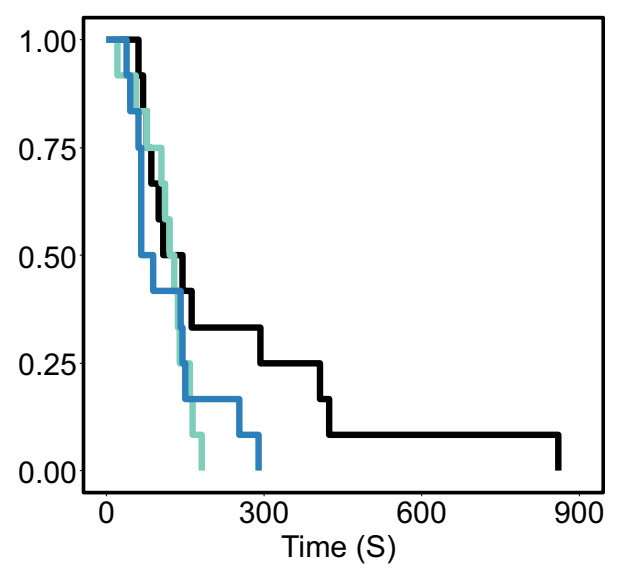

Treatment -0 NTU $=15$ NTU -30 NTU 
species: $\left.\mathrm{LRT}_{1,67}=63.77, P<0.001\right)$. Adding a quadratic term for turbidity, however, resulted in a significant interaction between turbidity and species (negative binomial GLM: species * poly(scale(turbidity)): $\left.\mathrm{LRT}_{2,64}=11.1, P=0.003\right)$. This interaction and the trends in Fig. 3a suggest that the number of feeding attempts by $O$. amphimelas was the greatest at the intermediate turbidity, but there was no such quadratic relationship in the trials with $O$. niloticus.

The lack of a significant interaction between species and turbidity revealed that turbidity had a similar effect on total food consumption in both species (negative binomial GLM: species * (scale(turbidity)): $\mathrm{LRT}_{1,66}=1.19, P=0.27$; Fig. $3 \mathrm{~b}$ ). As main effects, turbidity did not have a statistically significant effect on how many pellets were consumed (negative binomial GLM: (scale(turbidity)): $\mathrm{LRT}_{1,67}=3.29, P=0.06$ ). Species did have a significant effect, with $O$. amphimelas consuming less than $O$. niloticus (negative binomial GLM: species: $\left.\mathrm{LRT}_{1,66}=13.87, P<0.001\right)$.

\section{Experiment two}

For the likelihood of leaving the shelter for the first time, the interaction between species and turbidity was not significant, suggesting that turbidity had a similar effect in both species (CPH: hazard ratio $(\mathrm{HZ})=1.00, N=244, P=0.8)$. This likelihood did not differ between species $(O$. amphimelas versus O. niloticus: $\mathrm{CPH}$ : hazard ratio $(\mathrm{HZ})=0.58, N=244, P=$ $0.25)$. Turbidity did not have a statistically significant effect on the likelihood of leaving shelter (CPH: $\mathrm{HZ}=0.98, N=244$, $P=0.06$; Fig. 4a, b).

For the likelihood of crossing the midline for the first time, the interaction between species and turbidity was not significant, suggesting that turbidity had a similar effect in both species (CPH: $\mathrm{HZ}=1.00, N=244, P=0.75)$. The likelihood of crossing the midline did not differ significantly between species $(O$. amphimelas versus $O$. niloticus: $\mathrm{CPH}: \mathrm{HZ}=$
0.64, $N=244, P=0.13)$. Across both species, the likelihood of crossing the midline was lower in turbid (15 NTU) compared to clear water (CPH: $\mathrm{HZ}=0.96, N=244, P<0.001$; Fig. 4c, d).

The lack of a significant interaction between species and turbidity revealed that turbidity had a similar effect on the time outside shelter in both species (GLMM: species * (scale(turbidity)): $\left.\chi_{1}^{2}=0.33, P=0.56\right)$. As main effects the total time outside of the shelter did not differ between species (GLMM: species $\chi_{1}^{2}=0.53, P=0.46$; Fig. 5), and was lower in turbid water for both species (GLMM: scale (turbidity) $\chi_{1}^{2}$ $=20.31, P<0.001$; Fig. 5).

Spearman rank correlation coefficients showed interindividual consistency in the latency of $O$. amphimelas to first leave the shelter in the $15 \mathrm{NTU}$ treatment $\left(N=29, r_{S}=0.405\right.$, $P=0.029)$ but all other correlation coefficients were nonsignificant (Table 1), suggesting little support for strong consistent inter-individual variation in these species. We found no trend in boldness over time (estimate $=-0.008, \mathrm{SE}=0.02, P$ $=0.71$ ), suggesting that bolder individuals were not more likely to be caught first, and that haphazard sampling in regard to boldness was achieved in both experiments.

\section{Discussion}

Our results show that $O$. niloticus from aquaculture stock, which are those most likely to be introduced outside of the species' native range, started to feed more quickly, encountered more food items and consumed more in total than $O$. amphimelas bred from wild-caught parents. The latency to the first feeding attempt by $O$. amphimelas was unaffected by turbidity, while $O$. niloticus attempted to feed more readily when the water was turbid (15 and 30 NTU) than when it was clear (0 NTU). It is therefore surprising that the number of feeding attempts made by $O$. niloticus was not affected by
Fig. 3 The number of $\mathbf{a}$ feed attempts and $\mathbf{b}$ pellets consumed across turbidity treatments for both species, $O$. niloticus (white) and $O$. amphimelas (grey). The boxes represent the interquartile range with the middle line displaying the median. Each whisker represents the position of $50 \%$ of values outside of the interquartile range
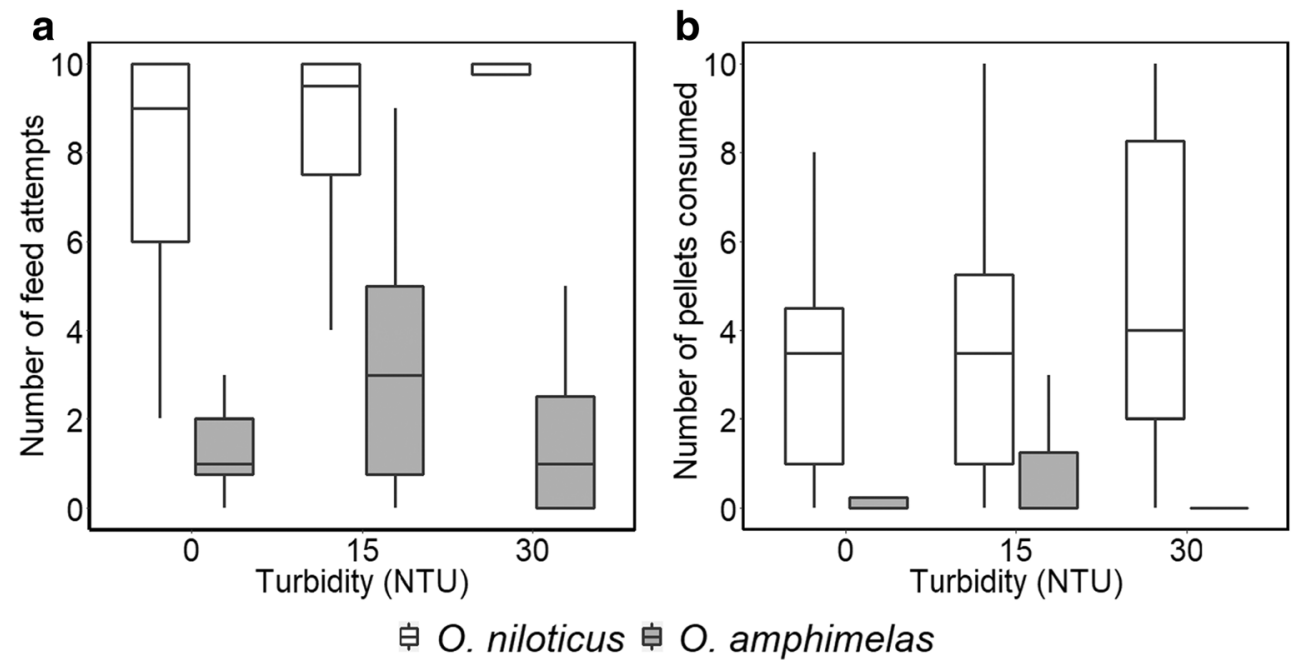

白 O. niloticus 白 $\mathrm{O}$. amphimelas 
Fig. 4 Kaplan-Meier event estimates displaying the proportion of individuals to remain in the shelter a $O$. amphimelas and $\mathbf{b} O$. niloticus and the proportion that did not cross the midline c $O$. amphimelas and $\mathbf{d}$ O. niloticus at 0 (solid) and 15 (dotted) NTU (level of turbidity) a

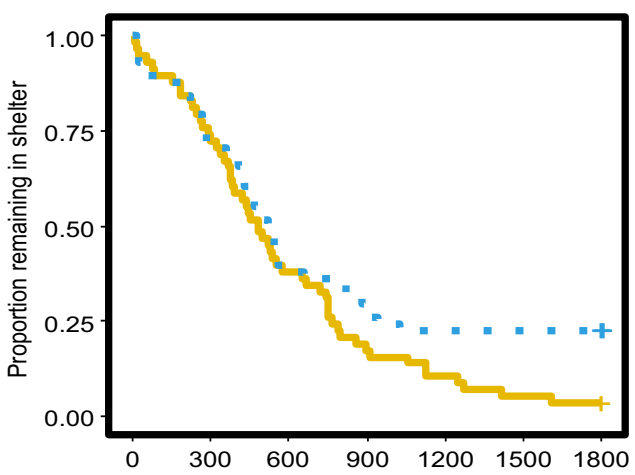

c

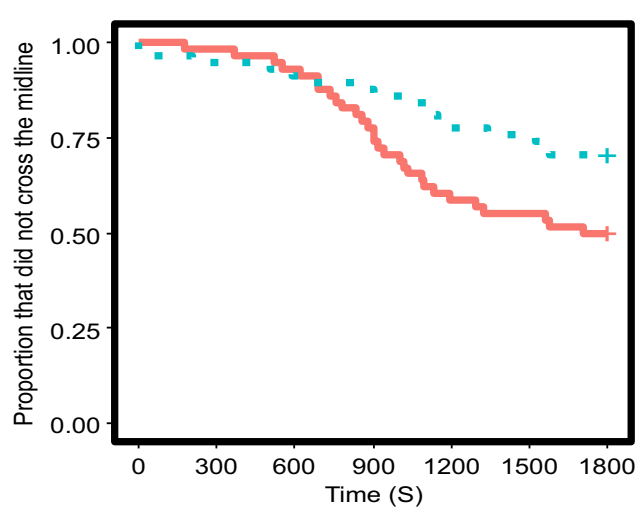

b

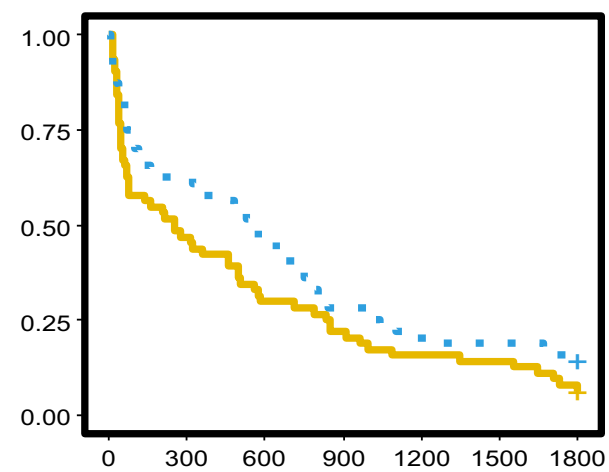

d

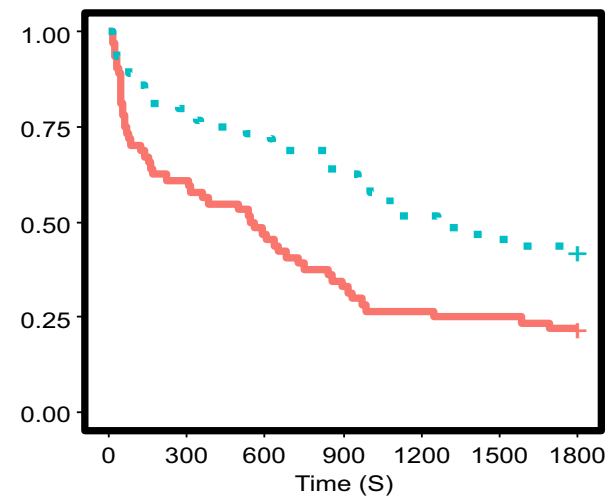

turbidity, suggesting that its propensity to initiate feeding may be higher in turbid water while its overall foraging success may be unaffected. Conversely, O. amphimelas had the highest number of feeding attempts at the intermediate turbidity of 15 NTU. This peak at intermediate turbidity was not found in the number of pellets consumed, which did not depend on turbidity for either species. In experiment

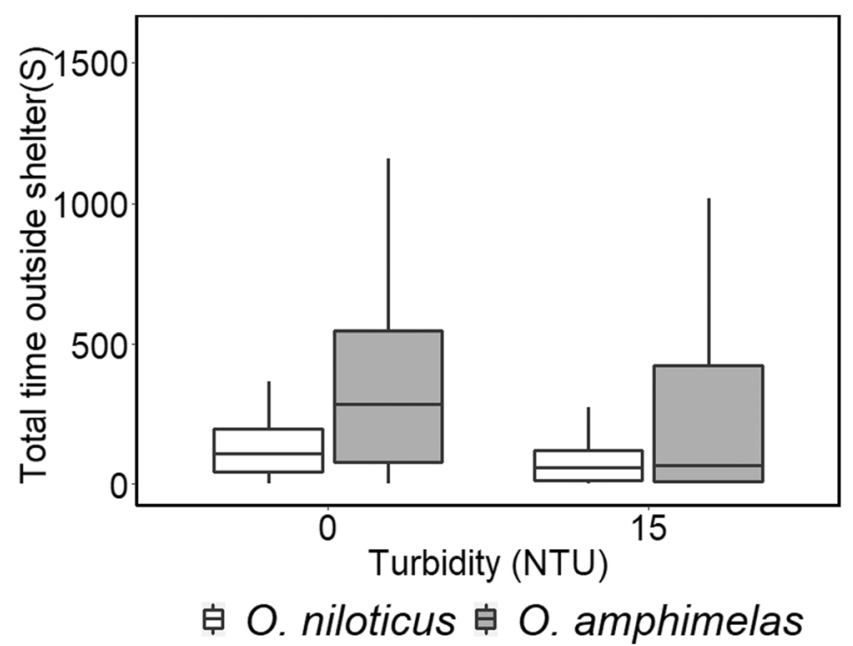

Fig. 5 Total time spent outside the shelter for $O$. niloticus (white) and $O$. amphimelas (grey) at 0 and $15 \mathrm{NTU}$ (level of turbidity). The median are horizontal lines within the boxes, the interquartile range (IQR) is the area within the boxes and the whiskers display the data within $1.5 \times \mathrm{IQR}$ two, the time taken to first leave the refuge was unaffected by turbidity. However, both species showed reduced risk taking in turbid conditions, where they were less likely to cross the midline of the arena and spent less time overall outside of the shelter in turbid conditions. This suggests that the difference between the species in their foraging response to turbidity in experiment one was not a result of differences in risk-taking tendency (boldness). There was little evidence of consistent differences between individuals (i.e. personality variation) in either clear or turbid water. $O$. amphimelas did display a positive correlation over different days in the latency to first leave the shelter in the turbid treatment, suggesting some indication of a consistent shybold continuum in turbid water. However, the lack of similar results across other behaviours makes this only suggestive in O. amphimelas and shows that personality variation in boldness was weak in this study compared to similar studies on other species (Brown et al. 2005; Biro et al. 2010; Bevan et al. 2018; Ehlman et al. 2019; SzopaComley et al. 2020a). While our investigation of boldness using refuge use in a novel environment has shown to be repeatable in other species (Bevan et al. 2018), stronger repeatability may have been observed in the presence of threatening stimuli specifically related to predation (Carter et al. 2010; Seltmann et al. 2012) or in the presence of food cues (Szopa-Comley et al. 2020b). 
Table 1 Spearman rank correlation coefficients $\left(r_{s}\right)$ for behaviours measured in two repeated trials in clear and turbid water. Twenty-nine $O$ amphimelas and 32 O. niloticus were tested. Significant values at 0.05 are shown in bold

\begin{tabular}{llll}
\hline & Turbidity (NTU) & $r_{s}$ & $p$ \\
\hline Latency to first leave shelter O. amphimelas & 0 & 0.044 & 0.817 \\
Latency to first leave shelter O. niloticus & 15 & $\mathbf{0 . 4 0 5}$ & $\mathbf{0 . 0 2 9}$ \\
& 0 & 0.037 & 0.836 \\
Latency to first cross midline O. amphimelas & 15 & 0.094 & 0.607 \\
Latency to first cross midline O. niloticus & 0 & 0.126 & 0.513 \\
& 15 & -0.292 & 0.123 \\
Total time outside shelter O. amphimelas & 0 & 0.088 & 0.629 \\
Total time outside shelter O. niloticus & 15 & 0.040 & 0.827 \\
& 15 & -0.201 & 0.293 \\
& 0 & 0.347 & 0.064 \\
& 15 & -0.143 & 0.432 \\
& & 0.088 & 0.629 \\
\hline
\end{tabular}

In our experiment, the fact that $O$. niloticus came from captive stock, while $O$. amphimelas were of wild origin, could be viewed as a potentially confounding variable, given that rearing history can effect risk taking and anti-predatory behaviours (Vilhunen and Hirvonen 2003). However, the majority of $O$. niloticus introductions result from intentional or accidental release of captive stock, rather than wild-type $O$. niloticus from their native range (Canonico et al. 2005). As a result, we feel that the experimental subjects used in this study accurately reflect the backgrounds of tilapia which would interact in situ. Thus, differentiating between the effects of rearing history and intrinsic differences between these two species is not necessary in order to make ecologically relevant conclusions about the impact of invasive species and increased turbidity.

Turbidity can have a negative impact on visual foragers by increasing foraging latency, reducing reaction distance and reducing attack success; however, our study showed that the feeding latency of $O$. niloticus was actually lower in the turbid treatment (Gregory and Levings 1996; Turesson and Brönmark 2007; Becker et al. 2016). O. niloticus may compensate for reduced visual information in turbid environments through increased activity and/or increasing reliance on another sensory modality. Past studies on $O$. niloticus demonstrate that it has an acute sense of olfaction that is used for foraging when vision is limited (Marusov and Kasumyan 2017). This could explain the increased propensity for $O$. niloticus to forage in turbid water, as species dependent on olfaction are not affected when foraging in turbid environments (Lunt and Smee 2015). However, direct comparisons of the sensory abilities of $O$. niloticus and native species including $O$. amphimelas are not available, so more information is needed in order to explore this hypothesis further. Compensatory factors such as increased sensory perception may explain why the time to first feed by $O$. amphimelas was not affected by turbidity but does not explain the more rapid time to feed in turbid water by $O$. niloticus, or the improvement in the number of feeding attempts by $O$. amphimelas from the clear to the intermediate turbidity treatment. The perception of risk by $O$. niloticus may have been lower in turbid water, possibly because turbidity was used as a refuge (Lehtiniemi et al. 2005; Ferrari et al. 2014). However, this was not supported by the results of experiment two, which showed reduced, rather than increased, risk taking in both species in turbid water. Our results may indicate that the response to turbidity may be highly context specific, having a different effect depending on the presence or absence of food; further experiments manipulating food odour cues as well as turbidity are needed.

For $O$. amphimelas, intermediate levels of turbidity may be advantageous when foraging but do not increase the motivation to initially forage as there was no effect of turbidity on the latency to first feed. This may allow $O$. amphimelas to forage in moderately turbid environments and potentially mitigate the effects of anthropogenic turbidity. However, our results indicate that this is only effective up to a certain threshold between 15 and 30 NTU. As previous work has indicated that turbidity between 26 and 141 NTU reduces the diversity and volume of stomach contents of fish species (Stuart-Smith et al. 2004), establishing at what range feeding efficiency is negatively affected is important and could help maintain populations of native species.

Considering that $O$. niloticus can play an active role in increasing turbidity through benthic foraging, its presence may initiate a reduction in quality of clear water habitats or encourage a further decline in those already degraded (Zhang et al. 2017). Our study shows that some aspects of feeding by O. niloticus increases in turbid water suggesting that habitat degradation driven by $O$. niloticus can be beneficial to its foraging. This follows previous studies that suggest that degraded habitats are favourable to invasive generalists allowing colonisation where native species decline (Marvier et al. 2004). If an introduction of $O$. niloticus leads to increased 
turbidity and so improves its feeding efficiency, it would become even more adept at foraging than native species. Thus, the introduction of $O$. niloticus could facilitate a positive feedback loop that results in it dominating competition with native species (Mainka and Howard 2010). This is especially relevant considering the foraging threshold of turbidity seen in $O$. amphimelas. If $O$. niloticus is introduced to an area where populations of $O$. amphimelas reside, it may increase the level of turbidity above this threshold potentially leading to the decline in foraging efficiency of $O$. amphimelas.

Our results demonstrate how the effects of turbidity differ between $O$. niloticus from aquaculture stock and wild-type $O$. amphimelas, a closely related species in the same genus. Some aspects of foraging in O. niloticus improved in turbid water, while high levels of turbidity constrained foraging in $O$. amphimelas by reducing the number of encounters with food. $O$. amphimelas did show improved foraging at intermediate turbidity levels, meaning that critical thresholds of turbidity may exist, past which native species could be outcompeted by $O$. niloticus which did not show reduced foraging efficiency even at the highest turbidity level. The increased foraging efficiency of $O$. niloticus over $O$. amphimelas in this experiment highlights how $O$. niloticus may present a threat to $O$. amphimelas and very likely other native cichlid species where it has been introduced, through exploitative competition for food resources. Our results suggest that turbidity could worsen these effects as $O$. niloticus has the potential to increase turbidity past critical thresholds where foraging efficiency declines in native species and while itself being unaffected by this degraded environment. However, we also found a number of foraging behaviours which were unaffected by turbidity or were improved at higher turbidity levels in both species. When food was not present, turbidity also increased the perception of risk in both species, which could influence predator prey interactions in situ; however, further experimental evidence is needed in order to expand on the potential impacts of this finding. Determining whether preventing habitats from becoming turbid is a potential management tool in combating the spread and negative effects of $O$. niloticus and similar invasive species would be a useful target for future studies and could provide policy makers with novel tools to combat invasive species. Further observations of $O$. niloticus under natural conditions are required to further understand how their behaviour can affect native species across its wide global distribution, with respect to foraging and other behaviours (Linde et al. 2008a, b; Salazar Torres et al. 2016; Zhang et al. 2017; Champneys et al. 2018, 2020).

Supplementary Information The online version contains supplementary material available at https://doi.org/10.1007/s00265-021-02984-8.

Acknowledgements We thank Professor George Turner from Bangor University for providing us with the $O$. amphimelas for this study. We would also like to thank the reviewers for their valuable feedback during the submission of this manuscript.

Funding TSC was funded by a NERC GW4+ FRESH CDT PhD studentship (NE/R011524/1). CCI was supported by the Natural Environment Research Council, grant number NE/P012639/1.

Data availability All data generated or analysed during this study are included in this published article (and its supplementary information files).

Code availability The code used in the current study is available from the corresponding author on reasonable request.

\section{Declarations}

Ethics approval and consent to participate All experimental procedures and housing conditions were in accordance with the ethical standards of the University of Bristol and ethical approval was granted by the University's Animal Welfare and Ethical Review Body (UB/18/067).

Consent for publication Not applicable.

Conflict of interest The authors declare no conflict of interest.

Open Access This article is licensed under a Creative Commons Attribution 4.0 International License, which permits use, sharing, adaptation, distribution and reproduction in any medium or format, as long as you give appropriate credit to the original author(s) and the source, provide a link to the Creative Commons licence, and indicate if changes were made. The images or other third party material in this article are included in the article's Creative Commons licence, unless indicated otherwise in a credit line to the material. If material is not included in the article's Creative Commons licence and your intended use is not permitted by statutory regulation or exceeds the permitted use, you will need to obtain permission directly from the copyright holder. To view a copy of this licence, visit http://creativecommons.org/licenses/by/4.0/.

\section{References}

Abrahams M, Kattenfeld M (1997) The role of turbidity as a constraint on predator-prey interactions in aquatic environments. Behav Ecol Sociobiol 40:169-174. https://doi.org/10.1007/s002650050330

Ajemian MJ, Sohel S, Mattila J (2015) Effects of turbidity and habitat complexity on antipredator behavior of three-spined sticklebacks (Gasterosteus aculeatus): Antipredator behavior in sticklebacks. Environ Biol Fish 98:45-55. https://doi.org/10.1007/s10641-0140235-x

Barrett JC, Grossman GD, Rosenfeld J (1992) Turbidity-induced changes in reactive distance of rainbow trout. Trans Am Fish Soc 121:437443. https://doi.org/10.1577/1548-8659(1992)121<0437:ticird >2.3. co;2

Bates D, Mächler M, Bolker BM, Walker SC (2015) Fitting linear mixedeffects models using lme4. J Stat Softw 67:1-48. https://doi.org/10. 18637/jss.v067.i01

Becker LJS, Brooks EM, Gabor CR, Ostrand KG (2016) Effects of turbidity on foraging behavior in the endangered fountain darter (Etheostoma fonticola). Am Midl Nat 175:55-63. https://doi.org/ 10.1674/amid-175-01-55-63.1 
Becker RA, Chambers JM, Wilks AR (1989) The New S Language. Wadsworth \& Brooks/Cole, Monterey

Bevan PA, Gosetto I, Jenkins ER, Barnes I, Ioannou CC (2018) Regulation between personality traits: Individual social tendencies modulate whether boldness and leadership are correlated. Proc R Soc B 285:20180829. https://doi.org/10.1098/rspb.2018.0829

Biro PA, Beckmann C, Stamps JA (2010) Small within-day increases in temperature affects boldness and alters personality in coral reef fish. Proc R Soc Lond B 277:71-77. https://doi.org/10.1098/rspb.2009. 1346

Biro PA, Dingemanse NJ (2009) Sampling bias resulting from animal personality. Trends Ecol Evol 24:66-67. https://doi.org/10.1016/j. tree.2008.11.001

Bolker B, R Core Team (2017) bbmle: Tools for General Maximum Likelihood Estimation. R package version 1.0.20, https://CRAN. R-project.org/package $=$ bbmle

Brown C, Gardner C, Braithwaite VA (2005) Differential stress responses in fish from areas of high- and low-predation pressure. J Comp Physiol B 175:305-312. https://doi.org/10.1007/s00360-005-0486-0

Brydges NM, Braithwaite VA (2009) Does environmental enrichment affect the behaviour of fish commonly used in laboratory work? Appl Anim Behav Sci 118:137-143. https://doi.org/10.1016/j. applanim.2009.02.017

Canonico GC, Arthington A, Mccrary JK, Thieme ML (2005) The effects of introduced tilapias on native biodiversity. Aquat Conserv Mar Freshwat Ecosyst 15:463-483. https://doi.org/10.1002/aqc.699

Carter AJ, Goldizen AW, Tromp SA (2010) Agamas exhibit behavioral syndromes: bolder males bask and feed more but may suffer higher predation. Behav Ecol 21:655-661. https://doi.org/10.1093/beheco/ $\operatorname{arq} 036$

Caves EM, Sutton TT, Johnsen S (2017) Visual acuity in ray-finned fishes correlates with eye size and habitat. J Exp Biol 220:15861596. https://doi.org/10.1242/jeb.151183

Chamberlain AC, Ioannou CC (2019) Turbidity increases risk perception but constrains collective behaviour during foraging by fish shoals. Anim Behav 156:129-138. https://doi.org/10.1016/j.anbehav.2019. 08.012

Chambers JM (2017) Linear models. In: Chambers JM, Hastie TJ (eds) Statistical models in S. Routledge, Boca Raton, pp 95-144

Champneys T, Castaldo G, Consuegra S, Garcia De Leaniz C (2018) Density-dependent changes in neophobia and stress-coping styles in the world's oldest farmed fish. R Soc Open Sci 5:181473. https://doi.org/10.1098/rsos.181473

Champneys T, Genner MG, Ioannou CC (2020) Invasive Nile tilapia dominates a threatened indigenous tilapia in competition over shelter. Hydrobiologia (published online, https://doi.org/10.1007/ s10750-020-04341-8)

Chivers DP, Al-Batati F, Brown GE, Ferrari MCO (2013) The effect of turbidity on recognition and generalization of predators and nonpredators in aquatic ecosystems. Ecol Evol 3:286-277. https://doi. org/10.1002/ece3.454

Davies-Colley RJ, Smith DG (2001) Turbidity, suspended sediment, and water clarity: A review. J Am Water Resour Assoc 37:1085-1101. https://doi.org/10.1111/j.1752-1688.2001.tb03624.x

Dearing JA, Jones RT (2003) Coupling temporal and spatial dimensions of global sediment flux through lake and marine sediment records. Glob Planet Chang 39:147-168. https://doi.org/10.1016/S09218181(03)00022-5

Dodds WK, Bouska WW, Eitzmann JL, Pilger TJ, Pitts KL, Riley AJ, Schloesser JT, Thornbrugh DJ (2009) Eutrophication of U. S. freshwaters: Analysis of potential economic damages. Environ Sci Technol 43:12-19. https://doi.org/10.1021/es801217q

Ehlman SM, Halpin R, Jones C, Munson A, Pollack L, Sih A (2019) Intermediate turbidity elicits the greatest antipredator response and generates repeatable behaviour in mosquitofish. Anim Behav 158: 101-108. https://doi.org/10.1016/j.anbehav.2019.10.006
Ehlman SM, Torresdal JD, Fraser DF (2020) Altered visual environment affects a tropical freshwater fish assemblage through impacts on predator-prey interactions. Freshw Biol 65:316-324. https://doi. org/10.1111/fwb. 13425

Engström-Öst J, Öst M, Yli-Renko M (2009) Balancing algal toxicity and turbidity with predation risk in the three-spined stickleback. J Exp Mar Biol Ecol 377:54-59. https://doi.org/10.1016/j.jembe.2009.06. 020

Erftemeijer PLA, Riegl B, Hoeksema BW, Todd PA (2012) Environmental impacts of dredging and other sediment disturbances on corals: A review. Mar Pollut Bull 64:1737-1765. https://doi.org/ 10.1016/j.marpolbul.2012.05.008

Eriksson BK, Sandström A, Isæus M, Schreiber H, Karås (2004) Effects of boating activities on aquatic vegetation in the Stockholm archipelago, Baltic Sea. Estuar Coast Shelf Sci 61:339-349. https://doi. org/10.1016/j.ecss.2004.05.009

Fabricius K, De'ath G, McCook L, Turak E, Williams DMB (2005) Changes in algal, coral and fish assemblages along water quality gradients on the inshore Great Barrier Reef. Mar Pollut Bull 51: 384-398. https://doi.org/10.1016/j.marpolbul.2004.10.041

Ferrari MCO, Elvidge CK, Jackson CD, Chivers DP, Brown GE (2010a) The responses of prey fish to temporal variation in predation risk: Sensory habituation or risk assessment? Behav Ecol 21:532-536. https://doi.org/10.1093/beheco/arq023

Ferrari MCO, Lysak KR, Chivers DP (2010b) Turbidity as an ecological constraint on learned predator recognition and generalization in a prey fish. Anim Behav 79:515-519. https://doi.org/10.1016/j. anbehav.2009.12.006

Ferrari MCO, Ranåker L, Weinersmith KL, Young MJ, Sih A, Conrad JL (2014) Effects of turbidity and an invasive waterweed on predation by introduced largemouth bass. Environ Biol Fish 97:79-90. https:// doi.org/10.1007/s10641-013-0125-7

Fox J, Weisberg S (2019) An R Companion to Applied Regression. Sage, Thousand Oaks, CA

Friard O, Gamba M (2016) BORIS: a free, versatile open-source eventlogging software for video/audio coding and live observations. Methods Ecol Evol 7:1325-1330. https://doi.org/10.1111/2041210X.12584

Gallardo B, Clavero M, Sánchez MI, Vilà M (2016) Global ecological impacts of invasive species in aquatic ecosystems. Glob Chang Biol 22:151-163. https://doi.org/10.1111/gcb.13004

García-Berthou E (2007) The characteristics of invasive fishes: What has been learned so far? J Fish Biol 71:33-55. https://doi.org/10.1111/j. 1095-8649.2007.01668.x

Granqvist M, Mattila J (2004) The effects of turbidity and light intensity on the consumption of mysids by juvenile perch (Perca fluviatilis L.). Hydrobiologia 514:93-101. https://doi.org/10.1023/B:hydr. $0000018210.66762 .3 \mathrm{~b}$

Gregory RS (1993) Effect of turbidity on the predator avoidance behaviour of juvenile chinook salmon (Oncorhynchus tshawytscha). Can J Fish Aquat Sci 50:241-246. https://doi.org/10.1139/f93-027

Gregory RS, Levings CD (1996) The effects of turbidity and vegetation on the risk of juvenile salmonids, Oncorhynchus spp., to predation by adult cutthroat trout, $O$. clarkii. Environ Biol Fish 47:279-288. https://doi.org/10.1007/BF00000500

Guthrie DM, Muntz WRA (1986) Role of vision in fish behaviour. In: Pitcher TJ (ed) The Behaviour of Teleost Fishes. Springer, Boston, MA

Harrison XA (2014) Using observation-level random effects to model overdispersion in count data in ecology and evolution. PeerJ 2: e616. https://doi.org/10.7717/peerj.616

Harvey BC, White JL (2008) Use of benthic prey by salmonids under turbid conditions in a laboratory stream. Trans Am Fish Soc 137: 1756-1763. https://doi.org/10.1577/t08-039.1

Hazelton PD, Grossman GD (2009a) Turbidity, velocity and interspecific interactions affect foraging behaviour of rosyside dace (Clinostomus 
funduloides) and yellowfin shiners (Notropis lutippinis). Ecol Freshw Fish 18:427-436. https://doi.org/10.1111/j.1600-0633. 2009.00359.x

Hazelton PD, Grossman GD (2009b) The effects of turbidity and an invasive species on foraging success of rosyside dace (Clinostomus funduloides ). Freshw Biol 54:1977-1989. https:// doi.org/10.1111/j.1365-2427.2009.02248.x

Hilton J, Phillips GL (1982) The effect of boat activity on turbidity in a shallow broadland river. J Appl Ecol 19:143-150. https://doi.org/10. 2307/2402998

Hinshaw JM (1985) Effects of illumination and prey contrast on survival and growth of larval yellow perch Perca flavescens. Trans Am Fish Soc 114:540-545. https://doi.org/10.1577/1548-8659(1985) $114<540$ :eoiapc $>2.0$. co; 2

Horppila J, Liljendahl-Nurminen A, Malinen T (2004) Effects of clay turbidity and light on the predator-prey interaction between smelts and chaoborids. Can J Fish Aquat Sci 61:1862-1870. https://doi. org/10.1139/F04-123

Ioannou CC, Dall SRX (2016) Individuals that are consistent in risktaking benefit during collective foraging. Sci Rep 6:33991. https:// doi.org/10.1038/srep33991

James G, Witten D, Hastie T, Tibshirani R (2000) An introduction to statistical learning: with applications in R. Springer, New York

Järvenpää M, Lindström K (2004) Water turbidity by algal blooms causes mating system breakdown in a shallow-water fish, the sand goby Pomatoschistus minutus. Proc R Soc Lond B 271:2361-2365. https://doi.org/10.1098/rspb.2004.2870

Järvenpää M, Lindström K (2011) Algal blooms decrease care but increase egg survival in a fish with paternal care. Behav Ecol Sociobiol 65:2023-2028. https://doi.org/10.1007/s00265-0111211-5

Johansen JL, Jones GP (2013) Sediment-induced turbidity impairs foraging performance and prey choice of planktivorous coral reef fishes. Ecol Appl 23:1504-1517. https://doi.org/10.1890/12-0704.1

Julien PY (1995) Erosion and sedimentation. Cambridge University Press, Cambridge

Kassambara A, Kosinski M, Biecek P (2019) Drawing Survival Curves using "ggplot2". R package version 0.4.5, https://CRAN.R-project. org/package $=$ survminer

Kemp P, Sear D, Collins A, Naden P, Jones I (2011) The impacts of fine sediment on riverine fish. Hydrol Process 25:1800-1821. https://doi. org/10.1002/hyp.7940

Kennard MJ, Arthington AH, Pusey BJ, Harch BD (2005) Are alien fish a reliable indicator of river health? Freshw Biol 50:174-193. https:// doi.org/10.1111/j.1365-2427.2004.01293.x

Leahy SM, McCormick MI, Mitchell MD, Ferrari MCO (2011) To fear or to feed: The effects of turbidity on perception of risk by a marine fish. Biol Lett 7:811-813. https://doi.org/10.1098/rsbl.2011.0645

Lehtiniemi M, Engström-Öst J, Viitasalo M (2005) Turbidity decreases anti-predator behaviour in pike larvae, Esox lucius. Environ Biol Fish 73:1-8. https://doi.org/10.1007/s10641-004-5568-4

Linde AR, Inácio AF, de Alburquerque C, Freire MM, Moreira JC (2008a) Biomarkers in an invasive fish species, Oreochromis niloticus, to assess the effects of pollution in a highly degraded Brazilian River. Sci Total Environ 339:186-192. https://doi.org/ 10.1016/j.scitotenv.2008.03.028

Linde AR, Izquierdo JI, Moreira JC, Garcia-Vazquez E (2008b) Invasive tilapia juveniles are associated with degraded river habitats. Aquat Conserv Mar Freshwat Ecosyst 18:891-895. https://doi.org/10. 1002/aqc. 928

Lunt J, Smee DL (2015) Turbidity interferes with foraging success of visual but not chemosensory predators. PeerJ 3:e1212. https://doi. org/10.7717/peerj. 1212

MacDougall AS, Turkington R (2005) Are invasive species the drivers or passengers of change in degraded ecosystems? Ecology 86:42-55. https://doi.org/10.1890/04-0669
Mainka SA, Howard GW (2010) Climate change and invasive species: Double jeopardy. Integr Zool 5:102-111. https://doi.org/10.1111/j. 1749-4877.2010.00193.x

Marusov EA, Kasumyan AO (2017) Feeding behavior and responsivity to food odors in Nile tilapia Oreochromis niloticus (Cichlidae) after chronic polisensory deprivation. J Ichthyol 57:747-752. https://doi. org/10.1134/S0032945217050113

Marvier M, Kareiva P, Neubert MG (2004) Habitat destruction, fragmentation, and disturbance promote invasion by habitat generalists in a multispecies metapopulation. Risk Anal 24:869-878. https://doi. org/10.1111/j.0272-4332.2004.00485.x

Meager JJ, Batty RS (2007) Effects of turbidity on the spontaneous and prey-searching activity of juvenile Atlantic cod (Gadus morhua). Philos Trans R Soc B 362:2123-2130. https://doi.org/10.1098/ rstb.2007.2104

Mohamed A-RM, Al-Wan MS (2020) Biological aspects of an invasive species of Oreochromis niloticus in the Garmat Ali River, Basrah, Iraq. J Agric Vet Sci 13:15-26

Mol JH, Ouboter PE (2004) Downstream effects of erosion from smallscale gold mining on the instream habitat and fish community of a small neotropical rainforest stream. Conserv Biol 18:201-214. https://doi.org/10.1111/j.1523-1739.2004.00080.x

Ormerod SJ, Dobson M, Hildrew AG, Townsend CR (2010) Multiple stressors in freshwater ecosystems. Freshw Biol 5:1-4. https://doi. org/10.1111/j.1365-2427.2009.02395.x

Orr JA, Vinebrooke RD, Jackson MC, Kroeker KJ, Kordas RL, MantykaPringle C, van den Brink PJ, de Laender F, Stoks R, Holmstrup M, Matthaei CD, Monk WA, Penk MR, Leuzinger S, Schäfer RB, Piggott JJ (2020) Towards a unified study of multiple stressors: Divisions and common goals across research disciplines. Proc R Soc B 287:20200421. https://doi.org/10.1098/rspb.2020.0421

Piggott JJ, Townsend CR, Matthaei CD (2015) Reconceptualizing synergism and antagonism among multiple stressors. Ecol Evol 5: 1538-1547. https://doi.org/10.1002/ece3.1465

Quesenberry NJ, Allen PJ, Cech JJ (2007) The influence of turbidity on three-spined stickleback foraging. J Fish Biol 70:965-972. https:// doi.org/10.1111/j.1095-8649.2007.01350.x

R core Team (2019) R: A language and environment for statistical computing. R Foundation for Statistical Computing, Vienna, Austria https://www.R-project.org/

Réale D, Reader SM, Sol D, McDougall PT, Dingemanse NJ (2007) Integrating animal temperament within ecology and evolution. Biol Rev 82:291-318. https://doi.org/10.1111/j.1469-185X.2007. 00010. $\mathrm{x}$

Salazar Torres G, Silva LHS, Rangel LM, Attayde JL, Huszar VLM (2016) Cyanobacteria are controlled by omnivorous filter-feeding fish (Nile tilapia) in a tropical eutrophic reservoir. Hydrobiologia 765:115-129. https://doi.org/10.1007/s10750-015-2406-y

Seltmann MW, Öst M, Jaatinen K, Atkinson S, Mashburn K, Hollmen T (2012) Stress responsiveness, age and body condition interactively affect flight initiation distance in breeding female eiders. Anim Behav 84:889-896. https://doi.org/10.1016/j.anbehav.2012.07.012

Shechonge A, Ngatunga BP, Bradbeer SJ, Day JJ, Freer JJ, Ford AGP, Kihedu J, Richmond T, Mzighani S, Smith AM, Sweke EA, Tamatamah R, Tyers AM, Turner GF, Genner MJ (2019) Widespread colonisation of Tanzanian catchments by introduced Oreochromis tilapia fishes: the legacy from decades of deliberate introduction. Hydrobiologia 832:235-253. https://doi.org/10.1007/ s10750-018-3597-9

Snow RA, Shoup DE, Porta MJ (2018) Effects of turbidity on prey selection and foraging rate of hatchery-reared juvenile tiger muskellunge. North Am J Fish Manag 38:487-492. https://doi.org/10. 1002/nafm. 10053

Stamps JA, Briffa M, Biro PA (2012) Unpredictable animals: Individual differences in intraindividual variability (IIV). Anim Behav 83: 1325-1334. https://doi.org/10.1016/j.anbehav.2012.02.017 
Stuart-Smith RD, Richardson AMM, White RWG (2004) Increasing turbidity significantly alters the diet of brown trout: A multi-year longitudinal study. J Fish Biol 65:376-388. https://doi.org/10.1111/j. 0022-1112.2004.00456.x

Sweka JA, Hartman KJ (2001) Effects of turbidity on prey consumption and growth in brook trout and implications for bioenergetics modeling. Can J Fish Aquat Sci 58:368-393. https://doi.org/10.1139/f00260

Sweka JA, Hartman KJ (2003) Reduction of reactive distance and foraging success in smallmouth bass, Micropterus dolomieu, exposed to elevated turbidity levels. Environ Biol Fish 67:341-347. https://doi. org/10.1023/A:1025835031366

Szopa-Comley AW, Donald WG, Ioannou CC (2020a) Predator personality and prey detection: inter-individual variation in responses to cryptic and conspicuous prey. Behav Ecol Sociobiol 74:70. https:// doi.org/10.1007/s00265-020-02854-9

Szopa-Comley AW, Duffield C, Ramnarine IW, Ioannou CC (2020b) Predatory behaviour as a personality trait in a wild fish population. Anim Behav 170:51-64. https://doi.org/10.1016/j.anbehav.2020. 10.002

Therneau TM (2015) A Package for Survival Analysis in S, version 2.38, https://CRAN.R-project.org/package=survival

Tuomainen U, Candolin U (2011) Behavioural responses to humaninduced environmental change. Biol Rev 86:640-657. https://doi. org/10.1111/j.1469-185X.2010.00164.x

Turesson H, Brönmark C (2007) Predator-prey encounter rates in freshwater piscivores: effects of prey density and water transparency. Oecologia 153:281-290. https://doi.org/10.1007/s00442-0070728-9

Utne-Palm AC (2002) Visual feeding of fish in a turbid environment: Physical and behavioural aspects. Mar Freshw Behav Physiol 35: 111-128. https://doi.org/10.1080/10236240290025644

Van De Meutter F, Stoks R, De Meester L (2005) The effect of turbidity state and microhabitat on macroinvertebrate assemblages: a pilot study of six shallow lakes. Hydrobiologia 542:379-390. https:// doi.org/10.1007/s10750-005-4941-4

Vander Zanden MJ, Olden JD (2008) A management framework for preventing the secondary spread of aquatic invasive species. Can J Fish Aquat Sci 65:1512-1522. https://doi.org/10.1139/F08-099

Vilhunen S, Hirvonen H (2003) Innate antipredator responses of Arctic charr (Salvelinus alpinus) depend on predator species and their diet. Behav Ecol Sociobiol 55:1-10. https://doi.org/10.1007/s00265003-0670-8

Vinyard GL, O'brien WJ (1976) Effects of light and turbidity on the reactive distance of bluegill (Lepomis macrochirus). J Fish Res Board Can 33:2845-2849. https://doi.org/10.1139/f76-342

Wenger AS, Johansen JL, Jones GP (2011) Suspended sediment impairs habitat choice and chemosensory discrimination in two coral reef fishes. Coral Reefs 30:879-887. https://doi.org/10.1007/s00338011-0773-z

Williams DMB (1982) Patterns in the distribution of fish communities across the Central Great Barrier Reef. Coral Reefs 1:35-43. https:// doi.org/10.1007/BF00286538

Wishingrad V, Musgrove AB, Chivers DP, Ferrari MCO (2015) Risk in a changing world: Environmental cues drive anti-predator behaviour in lake sturgeon (Acipenser fulvescens) in the absence of predators. Behaviour 152:635-652. https://doi.org/10.1163/1568539X00003246

Zhang X, Mei X, Gulati RD (2017) Effects of omnivorous tilapia on water turbidity and primary production dynamics in shallow lakes: implications for ecosystem management. Rev Fish Biol Fish 27: 245-254. https://doi.org/10.1007/s11160-016-9458-6

Publisher's note Springer Nature remains neutral with regard to jurisdictional claims in published maps and institutional affiliations. 